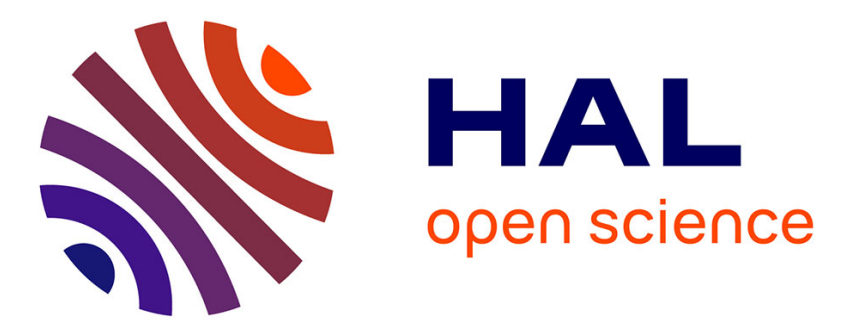

\title{
Probing the Fluorescence Dipoles of Single Cubic CdSe/CdS Nanoplatelets with Vertical or Horizontal Orientations
}

\author{
Fu Feng, Loan Thu Nguyen, Michel Nasilowski, Brice Nadal, Benoît \\ Dubertret, Agnès Maître, Laurent Coolen
}

\section{To cite this version:}

Fu Feng, Loan Thu Nguyen, Michel Nasilowski, Brice Nadal, Benoît Dubertret, et al.. Probing the Fluorescence Dipoles of Single Cubic CdSe/CdS Nanoplatelets with Vertical or Horizontal Orientations. ACS photonics, 2018, 5 ( 5), pp.1994-1999. 10.1021/acsphotonics.7b01475 . hal-01777981

\section{HAL Id: hal-01777981 \\ https: / hal.sorbonne-universite.fr/hal-01777981}

Submitted on 25 Apr 2018

HAL is a multi-disciplinary open access archive for the deposit and dissemination of scientific research documents, whether they are published or not. The documents may come from teaching and research institutions in France or abroad, or from public or private research centers.
L'archive ouverte pluridisciplinaire HAL, est destinée au dépôt et à la diffusion de documents scientifiques de niveau recherche, publiés ou non, émanant des établissements d'enseignement et de recherche français ou étrangers, des laboratoires publics ou privés. 


\title{
Probing the fluorescence dipoles of single cubic $\mathrm{CdSe} / \mathrm{CdS}$ nanoplatelets with vertical or horizontal orientations
}

Fu Feng ${ }^{1}$, Loan Thu NGuyen ${ }^{1,2}$, Michel Nasilowski ${ }^{3}$, Brice Nadal ${ }^{3}$, Benoît Dubertret ${ }^{3}$, Agnès Maître ${ }^{1}$, Laurent Coolen $^{1}$

${ }^{1}$ Sorbonne Universités, UPMC Univ Paris 06, CNRS-UMR 7588, Institut des NanoSciences de Paris, F-75005, Paris, France

${ }^{2}$ Institute of Materials Science, Vietnam Academy of Science and Technology, 18 Hoang Quoc Viet Road, Cau Giay Dist., 100000 Hanoi, Vietnam

${ }^{3}$ Laboratoire de Physique et d'Etude des Matériaux, ESPCI, UPMC Univ Paris 6, CNRS, 10 rue Vauquelin, Paris, France

\begin{abstract}
Measuring the orientation of a single fluorescent nano-emitter and obtaining emitters with a desired orientation is of highest importance for nanophotonics, especially in plasmonics where an emitting dipole close to a metallic surface will couple efficiently to plasmonic modes only if it is deposited vertically. Control of the orientation of a nano-object remains a challenge. Achieving vertical orientation, or having an information on the dipole orientation are key steps for efficient plasmonic excitation. We consider here cubic-shaped nanoplatelets with a thin CdSe core sandwiched in a thick CdS shell. By a combination of polarization measurement and radiation pattern Fourier analysis, we show that each single platelet behaves with excellent precision as a 2D dipole (sum of 2 orthogonal incoherent dipoles) and having only two possible orientations: either they lie horizontally on the substrate or they stand vertically on the edge. The cubic shape allows some platelets to deposit vertically so that they present a deterministic vertical dipole component.
\end{abstract}

Key words: single fluorescent emitter, nanoplatelet, emission polarization, radiation pattern

Colloidal semiconductor nanoparticles offer a wide range of applications in optics and opto-electronics. Spherical II-VI quantum dots are already commercially used for light-emitting diode (LED) displays and bioimaging labels. Elongated or tetrapod structures have been synthesized, and recently flat nanoplatelets with atomically controlled and monodisperse thickness ${ }^{1}$ have been produced, showing great potential for LEDs $^{2-3}$, photovoltaics ${ }^{4}$, lasers ${ }^{5-6}$ or photodetection ${ }^{7}$. For many applications, emission enhancement is sought thanks to a plasmonic structure. The coupling of the emitter to plasmonic modes is then strongly dependent on the dipole orientation and significantly enhanced for a dipole orthogonal to the metallic substrate $^{8-9}$. Moreover a precise understanding of the photophysics of these light emitters is necessary in order to optimize their synthesis protocol and optical properties. The radiation of the electron-hole pair states responsible for light emission is dependent on the quantum-confinement properties of these states, but may also involve dielectric antenna effects of the surrounding protection shell and environment, trapped charges in the vicinity of the emitter, ligands, etc. 
The emission from a nanorod ${ }^{10}$ or a dot-in-rod ${ }^{11}$ can be described as originating from a linear radiating dipole $^{12}$ ("1D dipole »). Spherical quantum dots, on the other hand, behave as an incoherent sum of 2 orthogonal dipoles («2D dipole ») $)^{13-15}$. Some results however suggest an intermediate behavior (sum of 1D and 2D dipoles) for dots-in-rods ${ }^{16}$ and quantum dots ${ }^{17}$, while $1 \mathrm{D}$-dipole emission has also been reported for quantum dots and attributed to ligand effects ${ }^{18}$.

While spherical quantum dots deposit with random orientations on substrates, a deterministic deposition of single emitters with horizontal dipole has been shown with either 1D (nanorod ${ }^{19}$ ) or 2D (dot-in-plate ${ }^{20}$ ) dipole natures. However, horizontal dipoles have very low interaction with the intense field close to the metallic surface and cannot benefit from surface-plasmonic enhancement as vertical ones do. Ensemble polarization measurements have shown that layers of fluorescent dye molecules with an overall vertical orientation could be deposited by using a liquid-crystal matrix ${ }^{21-23}$, with possibilities of orientation control by an applied voltage ${ }^{23}$ and of applications for luminescent solar concentrators ${ }^{21}$. However deposition with deterministic vertical dipole component has not to our knowledge been demonstrated at the single emitter level. Single-emitter radiation analysis is of importance in the context of fundamental photophysics study as well as for quantum optics experiments such as single-photon generation. Singleemitter manipulation by a plasmonic structure crucially depends on the presence of a vertical dipole.

In this paper, we demonstrate that by using single CdSe nanoplatelets with a thick CdS shell, it is possible to deposit some emitting dipoles orthogonal to metallic substrate which can benefit the most of plasmonic interaction. To this purpose, in order to evidence the orthogonal dipole, we use a method combining polarimetry and Fourier-plane analysis of a single nanoplatelet shell in order to obtain a precise determination of both the dipole nature (1D, 2D and potential existence of a third dipole) and the dipolar orientation.

In the first part of the paper, we present the experimental method. We then discuss the nature and orientation of the 2D emitting dipole. In the last part, we demonstrate that the contribution of a third linear dipole can be excluded, so that eventually some nanoplatelet emitters are shown to behave deterministically as $2 \mathrm{D}$ dipoles with the same vertical component 
(a)

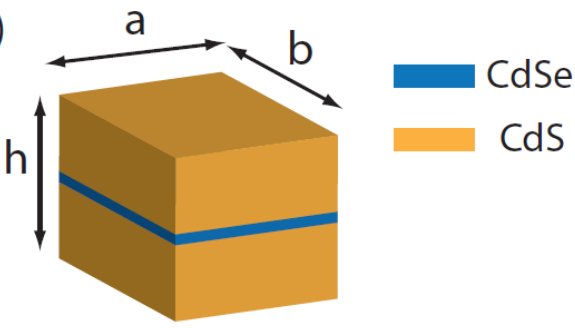

(c)

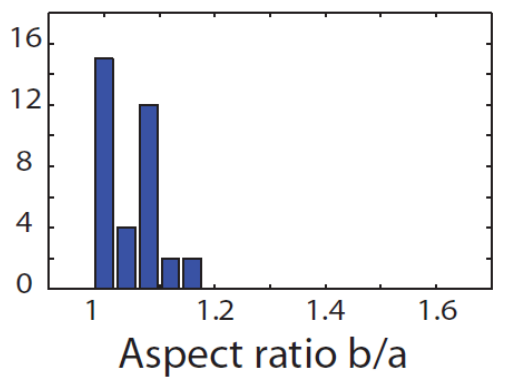

(b)

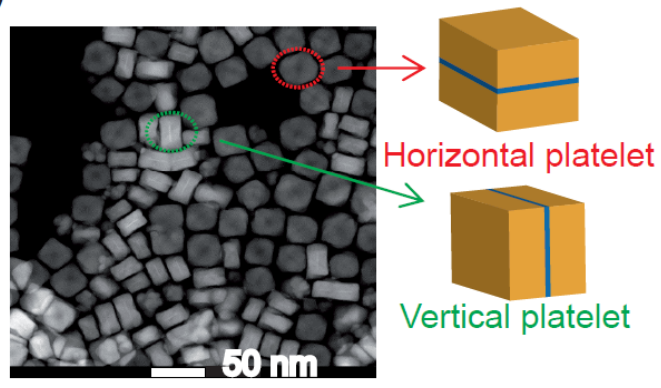

(d)

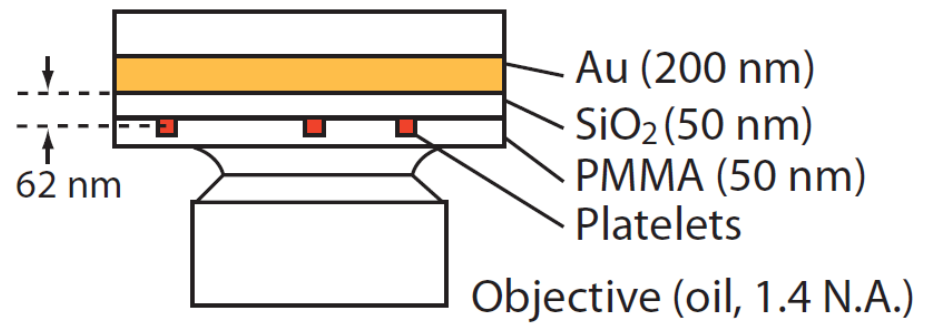

Figure1 (a) Schematic presentation of a cubic nanoplatelet. (b) TEM image of the nanoplatelets. (c) distribution of the aspect ratio b/a measured on the TEM image (b). (d) Schematic of the optical setup.

\section{Experimental protocol}

The nanoplatelets studied consist of a core CdSe square layer of $16 \times 16 \mathrm{~nm}^{2}$ lateral dimensions and $1.2 \mathrm{~nm}$ thickness, sandwiched between two layers of shell CdS which passivate its dangling bonds and improve its optical properties ${ }^{24}$ (fig. 1(a)). The nanoplatelets were synthesized following modifications of a protocol published $\mathrm{in}^{2}$ as described in the Supporting Information (S.I.). Unlike in previous reports ${ }^{25}$, we consider here thick shells of $8.5 \pm 1 \mathrm{~nm}$, so that the overall dimensions of the nanoplatelets are $18 \mathrm{~nm}$ in thickness and $29 \pm 3 \mathrm{~nm}$ lateral dimensions (with aspect ratio b/a between 1 and 1.18 where $a$ and $b$ are the lateral dimensions (fig 1(c)), which makes them closer to a cubic shape. Because of the cubic shape of these emitters, it is seen on transmission electron microscopy (TEM) images (fig. 1(b)) that the emitters can deposit both horizontally and vertically.

The cubic nanoplatelets are spin coated on a substrate with a density around 1 emitter per $10 \mu \mathrm{m}^{2}$. The substrate is a glass coverslip covered by a $200-\mathrm{nm}$ layer of gold with $50 \mathrm{~nm}$ of $\mathrm{SiO}_{2}$ on top (see S.I. for sample fabrication). $50 \mathrm{~nm}$ of PMMA are added on top of the sample in order to protect the sample from the environment (fig. 1(d)). An oil immersion objective is used (N.A. 1.4) so that for calculations the nanoplatelets can be considered embedded in a homogenous medium of index 1.5. The distance of the core or emitting dipole can be estimated at a distance of $62 \mathrm{~nm}$ (which accounts for the 50-nm silica and the shell thickness) from a thick gold layer. The platelets are excited by a blue laser beam ( $450 \mathrm{~nm}$ with 3.2 $\mu \mathrm{W}$ power) and the emission is collected by the same objective.

The radiation pattern and emission polarization are analyzed simultaneously to characterize the emission dipole nature and orientation from each single emitter. On the one hand the sample Fourier plane (rear 
focus plane of the objective) is imaged onto an EMCCD to perform the radiation pattern analysis: the image in the Fourier plane gives access to the distribution of the emitter's radiation into the $(\theta, \phi)$ angular coordinates (the z-axis being normal to the substrate). On the other hand, the emission polarization is analyzed by putting a rotating half wave plate followed by a polarized beam splitter (equivalent to a polarizer of angle $\alpha$ ) on the emission path. Two avalanche photodiodes are placed on the outputs of the cube and the normalized intensity $I=I_{1} /\left(I_{1}+I_{2}\right)$ is plotted as a function of $\alpha$. The setup was shown to modify polarization less than $5 \%$ (see discussion in S.I.).

The intensity of the emission of a given dipole analyzed by a rotating polarizer along angle $\alpha$ can be expressed in the form:

$$
I(\alpha)=I_{\min }+\left(I_{\max }-I_{\min }\right) \cos ^{2}\left(\Phi_{\max }-\alpha\right) \quad \text { (equation 1) }
$$

It was shown in ${ }^{12}$ that this curve can be used to obtain the $(\Theta, \Phi)$ angular coordinates of the emitting dipole orientation (note that $(\Theta, \Phi)$ coordinates are used to describe the dipole orientation while $(\theta, \phi)$ coordinates are used to describe the radiation pattern; $(\Theta, \Phi)$ is the orientation of the dipole for a 1D dipole and the orientation normal to the dipole plane for a 2D dipole). The in-plane orientation $\Phi$ is obtained as $\Phi=\Phi_{\max }$ for a $1 \mathrm{D}$ dipole and $\Phi=\Phi_{\max }+90^{\circ}$ for a 2D dipole. The degree of polarization $\delta$ is defined as $\delta=\left(I_{\max }-I_{\min }\right) /\left(I_{\max }+I_{\min }\right)$. Its value depends on the measurement conditions (substrate index, objective numerical aperture, 1D or 2D dipole) and on the zenithal (out-of-plane) orientation $\Theta$ of the dipole. Figure 2(a) plots, for the experimental conditions described before, the theoretical relation between the degree of polarization $\delta$ and orientation $\Theta$ following ${ }^{12}$. For $\Theta \sim 0^{\circ}$, the degree of polarization for both $1 \mathrm{D}$ and $2 \mathrm{D}$ dipoles is close to 0 as the system then presents axial symmetry. For $\Theta \sim 90^{\circ}$, the degree of polarization reaches its maximum ( 0.87 for a $2 \mathrm{D}$ dipole and 0.97 for a $1 \mathrm{D}$ dipole). Given this curve, the orientation $\Theta$ can be extracted from a measured value of $\delta$, however this requires that the 1D or $2 \mathrm{D}$ nature of the dipole is already known. The polarization analysis is not sufficient to determine both the dipole 1D/2D nature and its orientation.

The emission Fourier image can provide the necessary information to discriminate between $1 \mathrm{D}$ and $2 \mathrm{D}$ dipoles. Figure 2 (b) plots the Fourier image of the emission from a 1D or 2D dipole with $\Theta=0^{\circ}$ and $90^{\circ}$, calculated for the same measurement conditions following ${ }^{26}$. For the two orientations considered, the Fourier images are qualitatively very different for the 1D and 2D dipoles (let us note that the gold+silica layer substrate is a key element at this point: a glass substrate would lead to more similar Fourier patterns for the $1 \mathrm{D}$ and $2 \mathrm{D}$ cases) (see S.I.). It is thus possible to fully determine the emission dipole nature and its associated orientation by analyzing the emission polarization and radiation pattern simultaneously. In the next section, we apply this method to the case of the cubic nanoplatelets. 
(a)

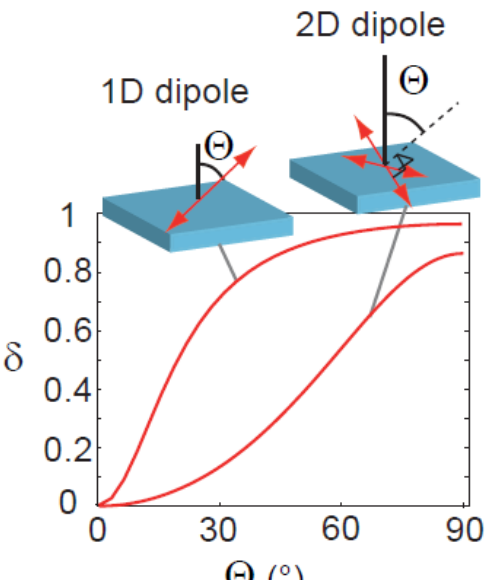

(b)
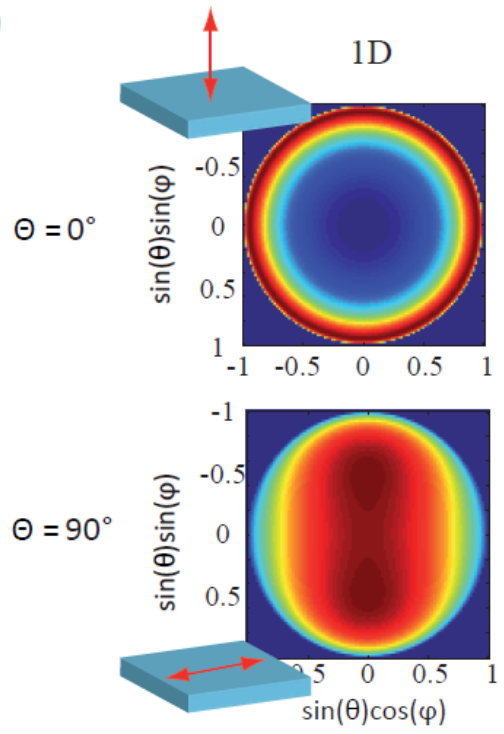

$2 \mathrm{D}$
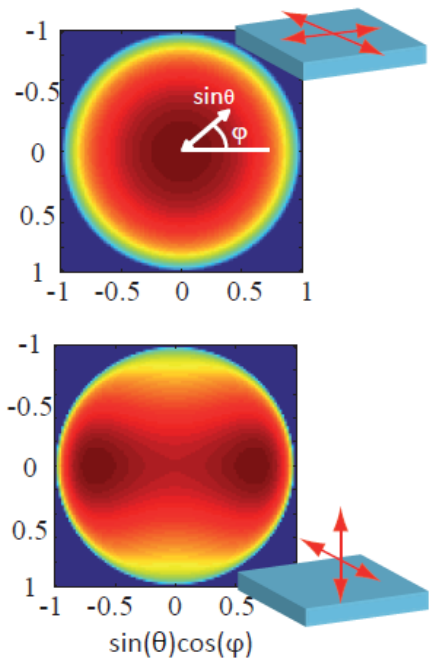

Figure 2 (a) Theoretical emission degree of polarization $(\delta)$ with respect to the dipole orientation $\Theta$ for 1D and $2 \mathrm{D}$ dipoles ( $\lambda=650 \mathrm{~nm}$, PMMA index 1.5, gold index $0.15+3.32 \mathrm{i}$, distance dipole-gold $62 \mathrm{~nm}$ ). (b) Theoretical radiation pattern for $1 \mathrm{D}$ or $2 \mathrm{D}$ dipoles with orientations 0 or $90^{\circ}$ (see S.I. for calculation details).

\section{Experimental results: horizontal and vertical 2D dipoles}

Figure 3 (a) shows the polarization analysis curve I( $\alpha$ ) for two typical nanoplatelets. The first platelet is representative of the main population of platelets, for which the degree of polarization is very close to 0 (a fit by eq. 1 gives for this platelet $\delta=0.08$ ). The second platelet belongs to a second population for which a much higher degree of polarization (here $\delta=0.79$ ) is obtained. Figure $3(c)$ shows in red (resp. green) a histogram of values of the degrees of polarization $\delta$ measured to the first (resp. second) population of platelets (note that the second population represents about $10 \%$ of the total platelets population). The emission degree of polarization is between 0 and 0.08 for the red population and between 0.77 to 0.88 for the green population. It is remarkable that no platelet was found with a degree of polarization between these two intervals: all platelets belonged to either one of the two very distinct populations.

As discussed in the first section, it is necessary to combine polarization with Fourier image analysis in order to fully characterize the emission dipole. For the first platelet, the very low degree of polarization suggests a 1D or 2D dipole with $\Theta \approx 0$ in either case. The measured Fourier image (fig. 3(b)) is qualitatively in agreement with the theoretical data calculated for a $2 \mathrm{D}$ dipole at $\Theta=0^{\circ}$ and very different from a 1D dipole at $\Theta=0^{\circ}$ (plotted in fig. 2(b)). From this data, figure 3(d) extracts the measured radiation patterns along directions $\phi=0$ and $90^{\circ}$ and compares them with the calculated radiation pattern from a 1D or 2D dipole at $\Theta=0^{\circ}$ (left and right figure respectively). These comparisons further confirm that the emission cannot be described by a 1D dipole but is on the other hand very well described by a 2D dipole at $\Theta=0^{\circ}$. Similar results were found for all nanoplatelets of the first population. The slightly non-zero value of $\delta$ for 
this $\Theta \approx 0$ population can be attributed to a slightly rectangular shape of the platelet (fig. 1(c)). Such spatial asymmetry induces a dielectric antenna effect due to the high optical index contrast between the nanoemitter material and the surrounding dielectric environment ${ }^{27}$ which can be at the origin of the small degree of polarization for horizontal platelets ${ }^{28}$.

For the second population, on the other hand, the high degree of population indicates a high value of $\Theta$ for either 1D or 2D dipole. The measured Fourier image for the second platelet (fig. 3(b)) is qualitatively in agreement with the theoretical Fourier image for a 2D dipole at $\Theta=90^{\circ}$, with the clear saddle shape which is less present for a 1D dipole (see fig. 2(b)). Further detailed comparison between the measurements and calculations (shown in fig. 3(d)) shows that the measured radiation pattern is significantly different from the calculated radiation pattern from a 1D dipole, but it is in good agreement with a 2D dipole of orientation $\Theta=90^{\circ}$ and $\Phi=137^{\circ}$. This in-plane orientation is in agreement with the value $\Phi_{\max }=47^{\circ}$ extracted from the polarization curve (fig. 3(a)) as $\Phi_{\max }=\Phi+90^{\circ}$ is expected for a 2D dipole. Similar results were found for all nanoplatelets of the second population and point to a second population of emitters behaving as $2 \mathrm{D}$ dipoles with $\Theta \approx 90^{\circ}$ orientation. The degree of polarization for this population is in the range 0.77-0.88 : the highest measured values are within measurement resolution from the theoretical $\delta\left(90^{\circ}\right)=0.87$. Following the approach developed $\mathrm{in}^{25}$, the lower values of $\delta$ for the second population can be explained by the slightly rectangular shape of the nanoplatelets.

This evidences that the emission from a cubic nanoplatelets can be described by a 2D dipole, with two distinct populations of orientations $\Theta \approx 0$ and $\Theta \approx 90^{\circ}$ due to the random deposition of the cubic platelets (while flat platelets with a thin CdS shell deposit only with the horizontal $\Theta=0$ orientation ${ }^{25}$ ).

The $2 \mathrm{D}$ nature of the dipole is in agreement with simple theoretical considerations for a thin quantum well: the large quantum confinement separates the heavy-hole state strongly from the upper light-hole state. The latter is then much higher in energy so that emission occurs only from the electronheavy-hole recombination ${ }^{1}$. As a result of the heavy-hole and electron band structure, this recombination has either \pm 1 or \pm 2 angular momentum ${ }^{29}$, of which only the degenerate \pm 1 transitions are optically allowed and contribute to the emission: the presence of another optically-forbidden state has been shown by lowtemperature decay measurements ${ }^{30}$. A 2D-dipole can thus be explained as an incoherent sum of \pm 1 transitions, which is equivalent to a sum of two orthogonal linear transitions. The 2D dipole is then expected to be parallel to the plane of the CdSe core. Alternatively, ensemble spectroscopic data suggest that a trion state (electron-hole pair recombining in presence of a third trapped resident charge) is responsible for the emission of nanoplatelets at low temperature ${ }^{31}$. Recent ensemble decay measurements in presence of a magnetic field ${ }^{32}$ have examined cubic nanoplatelets at low temperature : the allowed-state emission was then attributed to a negative trion recombination. The transition is then from the trion spin $\pm 3 / 2$ to the resident charge spin $\pm 1 / 2$, which also leads to allowed \pm 1 transitions corresponding to a 2D-dipole. Eventually, both exciton and trion emissions would be consistent with a 2D dipole parallel to the core platelet plane.

The presence of two distinct populations is then an indication of nanoplatelets deposited either horizontally $\left(\Theta=0^{\circ}\right)$ or on their side $\left(\Theta=90^{\circ}\right)$, as observed on the TEM images (fig. 1(b)). In this latter case, 
the 2D-dipole emitter exhibits a dipole which is vertical to the metallic surface, which permits a maximum interaction with plasmons enhanced fields.

(a)
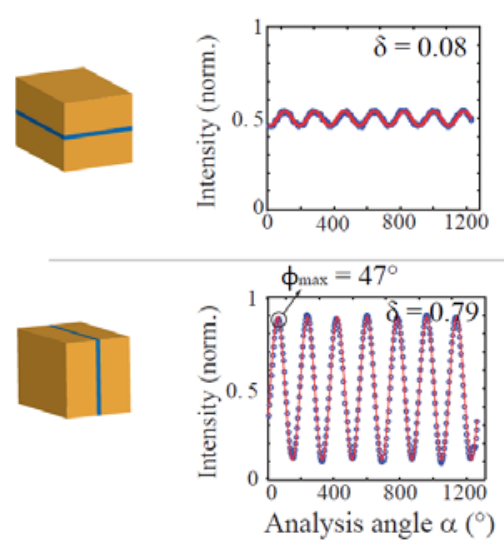

(c)

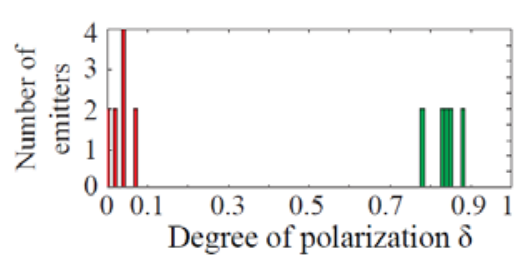

(d)
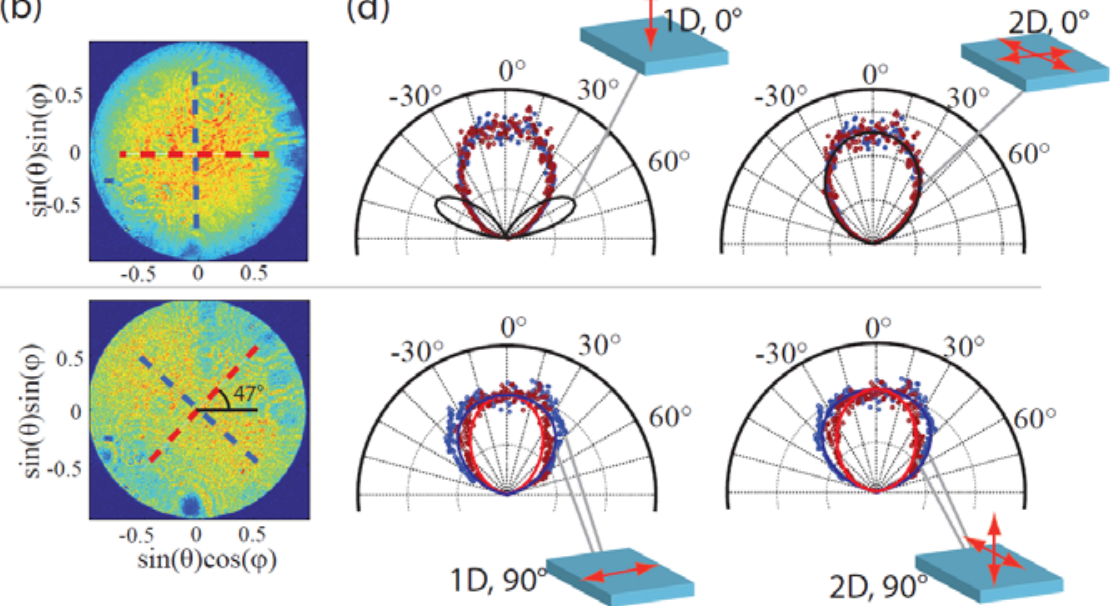

Figure 3 : Polarization analysis curves (a) and Fourier images (b) measured for two typical nanoplatelets. (c) Histogram of the measured degree of polarization for the two populations of platelets with almost-zero (red) and high (green) degree of polarization. (d) Dots: experimental radiation patterns of the two platelets, extracted from fig. $3(\mathrm{~b})$, along two $\phi$ directions (indicated in red and blue on fig. $3(\mathrm{~b})$ ) ; lines: tentative fits by the theoretical curve of a 1D (left) or 2D (right) dipole.

\section{Further analysis of the dipolar transition}

For the moment, we have considered that the nanoplatelet dipole could be modeled either by pure 1D and 2D dipoles, and finally excluded the 1D case. However, for other semiconductor nanoparticles, it has been reported that the emission might be a sum of a 2D dipole and a third orthogonal 1D dipole with different oscillator strengths ${ }^{16-17}$. However these discussions relied either on the assumption that the dipole orientation was known ${ }^{16}$ or on very delicate quantitative analysis of the polarization and defocused image ${ }^{17}$. In the following section, we will demonstrate that, for these nanoplatelets, we can exclude the emission from a third dipole to less than $5 \%$.

Assuming the presence of a third dipole $d_{3}$ with a smaller oscillation strength, orthogonal to the 2D-dipole plane, we define the factor $\varepsilon$ to describe the relation of oscillation strengths for these three dipoles by $\frac{\left|d_{3}\right|^{2}}{\left|d_{1}\right|^{2}+\left|d_{2}\right|^{2}}=\varepsilon$ (with $\mathrm{d}_{1}=\mathrm{d}_{2}$ here for a 2D dipole). The relation between the degree of emission polarization and the factor $\varepsilon$ in the case $\Theta=90^{\circ}$ is plotted in Figure 4(a). When $\varepsilon=0$, the degree of emission 
polarization in this case is thus 0.87 as shown in Figure 2(a) (2D dipole). When $\varepsilon=0.5$, all three dipoles have equal oscillator strengths and the emission is unpolarized ("3D dipole"). The measured degrees of emission of the green population (histogram of fig. $3(c)$ ) are just in the range $0.77-0.88$, which corresponds theoretically to $\varepsilon=0-0.03$. For a value of $\varepsilon=0.05$, even by taking into account the elongated shape of the platelets, the theoretical value of the degree of polarization gets to 0.74 , which remains below the experimental values.

We now consider the Fourier image data, for instance for the same emitter as in Figure 3(c). Figure 4(b-e) presents fits of the measured radiation patterns (same as fig. 3(d)) with the theoretical calculation for different values of factor $\varepsilon$. The value $\varepsilon=0$ gives the best fit of the data, and $\varepsilon=0.05$ is also very close. Starting from $\varepsilon=0.1$, and more clearly at $\varepsilon=0.25$, a difference between theory and experiment begins to be distinguishable. To conclude this study, the contribution from the third dipole $\mathrm{d}_{3}$ for a nanoplatelet can be deduced from both measurements to be very small: less than $10 \%$ from Fourier images and even less than $5 \%$ from polarization data. The method thus provides an excellent precision for the dipole analysis of nanoemitters. The very fine control over the nanoplatelets synthesis provides emitters with very good agreement to theory.

(a)

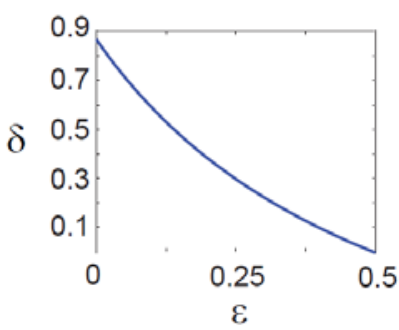

(b) $\varepsilon=0.25$

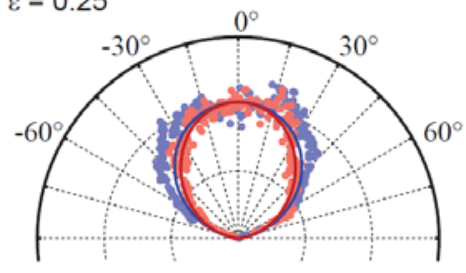

(c) $\varepsilon=0.1$

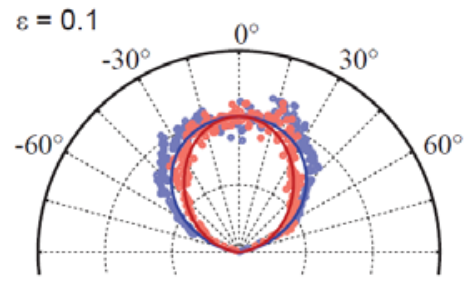

(d) $\varepsilon=0.05$

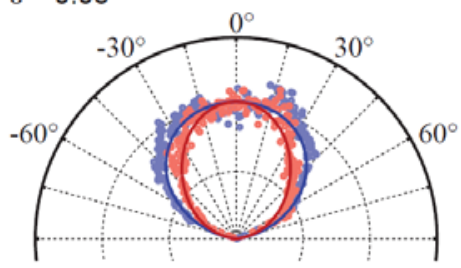

(e) $\varepsilon=0$

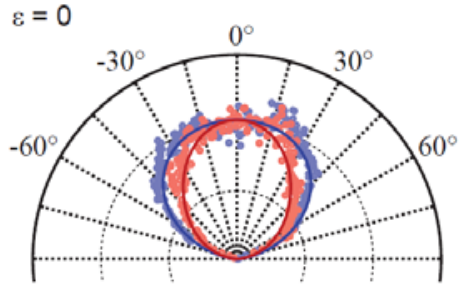

Figure 4 (a). (a) Calculated relation between emission degree of polarization $\delta$ and factor $\varepsilon$. (b-e) Comparison between the measured emission pattern along $\phi=\Phi_{\max }$ (red dots) and $\phi=\Phi_{\max }+90^{\circ}$ (blue dots) and the calculated emission pattern from an incoherent sum of a 2D dipole and a 1D dipole at $\Theta=$ $90^{\circ}, \Phi=\Phi_{\max }+90^{\circ}$ for different values of $\varepsilon$.

\section{Conclusion}

It has been demonstrated that by combining the emission polarization analysis and radiation pattern analysis, the emission dipole nature and orientation of a single nanoemitter can be fully determined. The excellent agreement between the experiment data and the calculations further demonstrate the relevance of the method developed here. We demonstrated that the emission from cubic-shaped (thick-shell) nanoplatelet present perfect (better than $95 \%$ ) 2D-dipole behavior. Thanks to the independent analysis 
of single emitters and to the combination of polarimetric and emission diagram measurement, we have demonstrated two single different orientations of deposition of the platelets; either horizontally or vertically. The occurrence of vertical platelets deposition, due to their cubic shape, allows for a vertical dipole component which can be of major interest for efficient surface plasmon coupling and emission optimization. This latter feature remains for other conventional emitters very challenging and even for spherical nanocrystals the occurrence of dipoles perpendicular to the surface by spin coating deposition is very low. Therefore, the use of cubic colloidal nanoplatelets, offering just 2 orientations for the dipoles, and quite high probability to obtain vertical orientation, is an important improvement for plasmonics. The easy operation of such colloidal nanoplatelets, their optical quality, and their semi deterministic orientation make them appropriate objets in photonic devices.

\section{Acknowledgements}

This work was supported by the Agence Nationale de la Recherche (project JCJC 12-JS04-0011-01 PONIMI), the Centre de Compétence Nanosciences Ile-de-France (Patch project) and by the CNRS program Platon (PICS 6456). The authors would like to thank their coworkers at INSP: Catherine Schwob and Jean-Marc Frigerio for fruitful discussions, Willy Daney de Marcillac for his help on the microscopy setup, Francis Breton for the setup interface and Loïc Becerra and Mélanie Escudier for the substrate preparation.

\section{Supporting information}

Nanoplatelets synthesis and spectral properties; micro-photoluminescence setup, polarization and Fourier plane measurements; sample preparation; theoretical radiation pattern calculations.

\section{Reference}

1. S. Ithurria, M. D. Tessier, B. Mahler, R. P. S. M. Lobo, B. Dubertret and Al. L. Efros, Colloidal nanoplatelets with two-dimensional electronic structure, Nat. Materials. 2011, 10, 936.

2. Z. Chen, B. Nadal, B. Mahler, H. Aubin and B. Dubertret, Quasi-2D colloidal semiconductor nanoplatelets for narrow electroluminescence, Adv. Funct. Mater. 2014, $24,295$.

3. N. Hasanov, V. K. Sharma, P. Martinez, S. T. Tan and H. V. Demir, Critical role of CdSe nanoplatelets in color-converting CdSe/ZnS nanocrystals for InGaN/GaN light-emitting diodes, Optics Lett. 2016, 41, 2883.

4. B. Guzelturk, F. Menk, K. Philipps, Y. Kelestemur, M. Olutas, R. Zentel and H. V. Demir, Colloidal nanoplatelet/conducting polymer hybrids : excitonic and material properties, J. Phys. Chem. C. 2016, 120, 3573.

5. M. Li, M. Zhi, H. Zhu, W.-Y. Wu, Q.-H. Xu, M. H. Jhon and Y. Chan, Ultralow-threshold multiphotonpumped lasing from colloidal nanoplatelets in solution, Nature Comm. 2015, 6, 8513.

6. C. She, I. Fedin, D. S. Dolzhnikov, P. D. Dahlberg, G. S. Engel, R. D. Schaller and D. V. Talapin, Red, yellow, green and blue amplified spontaneous emission and lasing using colloidal CdSe nanoplatelets, ACS Nano. 2015, 9, 9475.

7. E. Lhuillier, J.-F. Dayen, D. O. Thomas, A. Robin, B. Doudin and B. Dubertret, Nanoplatelets bridging a nanotrench : a new architecture for photodetectors with increased sensitivity, Nano Lett. 2015, 15, 1736. 
8. C. Vion, P. Spinicelli, L. Coolen, J.-M. Frigerio, J. P. Hermier and A. Maître, Controlled modification of single colloidal CdSe/ZnS nanocrystal fluorescence through interactions with a gold surface, Optics Express. 2010, 18, 7440.

9. C. Belacel, B. Habert, F. Bigourdan, F. Marquier, J-P. Hugonin, S. Michaelis de Vasconcellos, X. Lafosse, L. Coolen, C. Schwob, C. Javaux, B. Dubertret, J-J. Greffet, P. Senellart and A. Maître, Controlling spontaneous emission with plasmonic optical patch antennas, Nanoletters. 2013, 13 1516.

10. X. Chen, A. Nazzal, D. Goorskey, M. Xiao, Z. A. Peng and X. Peng, Polarization spectroscopy of single CdSe quantum rods, Phys. Rev. B. 2001, 64, 245304.

11. D. V. Talapin, R. Keoppe, S. Götzinger, A. Kornomski, J. M. Lupton, A. L. Rogach, O. Benson, J. Feldmann and $\mathrm{H}$. Weller, Highly emissive colloidal $\mathrm{CdSe} / \mathrm{CdS}$ heterostructures of mixed dimensionality, Nano Lett. 2003, 3, 1677.

12. C. Lethiec, F. Pisanello, L. Carbone, A. Bramati, L. Coolen and A. Maître, Polarimetry-based analysis of dipolar transitions of single colloidal CdSe/CdS dot-in-rods, New J. Phys. 2014, 16, 093014.

13. S. A. Empedocles, R. Neuhauser and M. G. Bawendi, Three-dimensional orientation measurements of symmetric single chromophores using polarization microscopy, Nature. 1999, 399, 126.

14. X. Brokmann, L. Coolen, M. Dahan and J.-P. Hermier, Measurement of the radiative and nonradiative decay rates of single CdSe nanocrystals through a controlled modification of their spontaneous emission, Phys. Rev. Lett. 2004, 93, 107403.

15. C. Lethiec, J. Laverdant, H. Vallon, C. Javaux, B. Dubertret, J.-M. Frigerio, C. Schwob, L. Coolen and A. Maître, Measurement of three-dimensional dipole orientation of a single fluorescent nanoemitter by emission polarization analysis, Phys. Rev. X 4. 2013, 021037.

16. S. Vezzoli, M. Manceau, G. Leménager, Q. Glorieux, E. Giacobino, L. Carbone, M. De Vittorio and A. Bramati, Exciton fine structure of $\mathrm{CdSe} / \mathrm{CdS}$ nanocrystals determined by polarization microscopy at room temperature, ACS Nano. 2015, 9, 7992.

17. A. Cyphersmith, K. Early, A. Maksov, J. Graham, Y. Wang and M. Barnes, Disentangling the role of linear transition dipole in band-edge emission from single CdSe/ZnS quantum dots : combined linear anisotropy and defocused radiation pattern imaging, Appl. Phys. Lett. 2010, 97, 121915.

18. K. T. Early, K. McCarthy, M. Y. Odoi, P. K. Sudeep, T. Emrick and M. D. Barnes, Linear dipole behavior in single CdSe-oligo(phenylene vinylene) nanostructures, ACS Nano. 2009, 3, 453.

19. I.Hadar, G.B.Hitin, A.Sitt, A.Faust and U.Banin, Polarization properties of semiconductor nanorod heterostructures: from single particles to the ensemble. J. Phys. Chem. Lett. 2013, 4, 502.

20. E. Cassette, B. Mahler, J.-M. Guigner, G. Patriarche, B. Dubertret and T. Pons, Colloidal CdSe/CdS dot-in-plate nanocrystals with 2D-polarized emission, ACS Nano. 2012, 6, 6741.

21. C. L. Mulder, P. D. Reusswig, A. M. Velasquez, H. Kim, C. Rotschild and M. A. Baldo, Dye alignment in luminescent solar concentrators : I. Vertical alignment for improved waveguide coupling, Opt. Express. 2010, 18, A79.

22. M. G. Debije, Solar energy collectors with tunable transmission, Adv. Funct. Mater. 20, 1498 (2010),

23. R. MacQueen, Y. Y. Cheng, R. G. C. R. Clady and T. W. Schmidt, Towards an aligned luminophore solar concentrator, Opt. Express. 2010, 18, A161.

24. M.D.Tessier, B.Mahler, B.Nadal, S.Pedetti, H.Heuclin and B.Dubertret, Spectroscopy of colloidal semiconductor core/shell nanoplatelets with high quantum yield, Nano Lett. 2013, 13, 3321.

25. B. Mahler, B. Nadal, C. Bouet, G. Patriarche and B. Dubertret, Core/shell colloidal semiconductor nanoplatelets, J. Am. Chem. Soc. 2012, 134, 18591. 
26. W. Lukosz, Light emission by magnetic and electric dipoles close to a plane dielectric interface. III. Radiation patterns of dipoles with arbitrary orientation, J. Opt. Soc. Am. 1979, 69, 1495.

27. A. V. Rodina and Al. L. Efros, Effect of dielectric confinement on optical properties of colloidal nanostructures, J. Exp. Theor. Phys. 2016, 122, 554.

28. F. Feng, L. T. N'Guyen, M. Nasilowski, B. Nadal, B. Dubertret, L. Coolen and A. Maître, Consequence of shape elongation on emission asymmetry for colloidal $\mathrm{CdSe} / \mathrm{CdS}$ nanoplatelets, to be published in Nano Research,

29. I. Chung, K. T. Shimizu and M. G. Bawendi, Room temperature measurements of the 3D orientation of single CdSe quantum dots using polarization microscopy, Proc. Nat. Ac. Sc. 2003, 100, 405.

30. L. Biadala, F. Liu, M. D. Tessier, D. R. Yakovlev, B. Dubertret and M. Bayer, Recombination dynamics of band edge excitons in quasi-two-dimensional CdSe nanoplatelets, Nano Lett. 2014, 14, 1134.

31. E. V. Shornikova, L. Biadala, D. R. Yakovlev, V. F. Sapega, Y. G. Kusrayev, A. A. Mitioglu, M. V. Ballottin, P. C. M. Christianen, V. V. Belykh, M. V. Kochiev, N. N. Sibeldin, A. A. Golovatenko, A. V. Rodina, N. A. Gippius, A. Kuntzmann, Y. Jiang, M. Nasilowski, B. Dubertret and M. Bayer, Adressing the exciton fine structure in colloidal nanocrystals : the case of CdSe nanoplatelets, Nanoscale. 2018, 10, 646.

32. E. V. Shornikova, L. Biadala, D. R. Yakovlev, D. Feng, V. F. Sapega, N. Flipo, A. A. Golovatenko, M. A. Semina, A. V. Rodina, A. A. Mitioglu, M. V. Ballottin, P. C. M. Christianen, Y. G. Kusrayev, M. Nasilowski, B. Dubertret and M. Bayer, Electron and hole g-factors and spin dynamics of negatively charged excitons in CdSe/CdS colloidal nanoplatelets with thick shells, Nano Lett. 2018, 18, 373. 